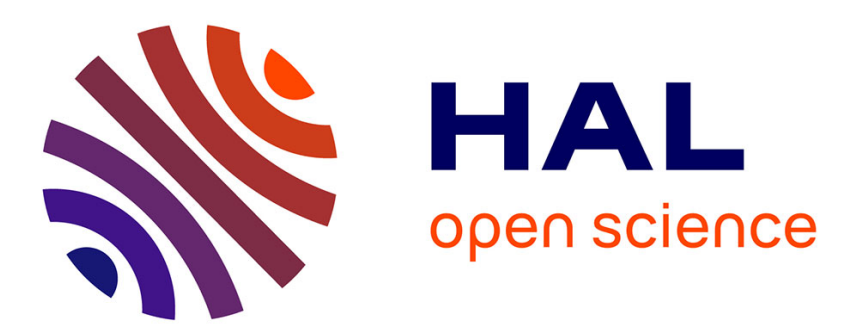

\title{
Propriétés électrochimiques d'électrodes monocristallines de tellurure de zinc non intentionnellement dopées
}

\author{
J.L. Sculfort
}

\section{- To cite this version:}

J.L. Sculfort. Propriétés électrochimiques d'électrodes monocristallines de tellurure de zinc non intentionnellement dopées. Revue de Physique Appliquée, 1979, 14 (9), pp.829-835. 10.1051/rphysap:01979001409082900 . jpa-00244669

\section{HAL Id: jpa-00244669 https://hal.science/jpa-00244669}

Submitted on 1 Jan 1979

HAL is a multi-disciplinary open access archive for the deposit and dissemination of scientific research documents, whether they are published or not. The documents may come from teaching and research institutions in France or abroad, or from public or private research centers.
L'archive ouverte pluridisciplinaire HAL, est destinée au dépôt et à la diffusion de documents scientifiques de niveau recherche, publiés ou non, émanant des établissements d'enseignement et de recherche français ou étrangers, des laboratoires publics ou privés. 


\title{
Propriétés électrochimiques d'électrodes monocristallines de tellurure de zinc non intentionnellement dopées
}

\author{
J. L. Sculfort \\ Laboratoire d'électrochimie interfaciale du C.N.R.S., \\ 1, place Aristide-Briand, 92190 Meudon Bellevue, France
}

(Reçu le 22 décembre 1978, révisé le 8 juin 1979, accepté le 11 juin 1979)

\begin{abstract}
Résumé. - Ce travail concerne l'étude de l'impédance de l'interface d'une électrode monocristalline de $\mathrm{ZnTe}$ et d'un électrolyte indifférent de $\mathrm{pH}$ variable.

Nous avons montré l'existence de niveaux profonds attribués au zinc et d'états localisés en surface qui impliquent une variation très importante des éléments de l'impédance en fonction de la fréquence de la perturbation alternative.

Le nombre de porteurs majoritaires a été évalué. La position des niveaux d'énergie est donnée en fonction de l'index de la face cristallographique et de la nature des anions à l'interface.
\end{abstract}

\begin{abstract}
In this work, impedance measurements of single crystals $\mathrm{ZnTe}$ electrodes in indifferent electrolytes (with different $\mathrm{pH}$ ) has been studied.

The observed frequency dependence of the capacitance and resistance can be attributed to deep levels due to zinc and surface states. The number of majority carriers has been evaluated. Positions of energy levels is obtained in relation to the nature of anions at the interface.
\end{abstract}

L'étude des jonctions existant entre une électrode semiconductrice et un électrolyte est d'un intérêt fondamental pour comprendre le rôle de la structure électronique du solide dans les réactions d'électrode. $\mathrm{La}$ vitesse des réactions de transfert de charges à l'électrode est fonction du nombre de porteurs de charge et de la position des niveaux d'énergie du solide et de la solution.

Notre choix s'est porté sur l'étude des électrodes monocristallines semiconductrices à large bande interdite $\left(E_{\mathrm{G}} \geqslant 2 \mathrm{eV}\right)$. Ce travail concerne plus particulièrement le comportement de jonctions semiconducteur-électrolyte avec des électrodes monocristallines de tellurure de zinc $(\mathrm{ZnTe})\left(E_{\mathrm{G}}=2,26 \mathrm{eV}\right.$ à $\left.300 \mathrm{~K}\right)$.

1. Préparation des électrodes. - L'utilisation de ce matériau nécessite un grand nombre d'opérations préalables :

- la cristallogenèse de ce composé (II/VI) à structure c.f.c. analogue à la blende, est réalisée en solution en léger excès de tellure par la méthode de Bridgman [1]. Les cristaux non intentionnellement dopés présentent une conduction de type $p$. L'homogénéité des cristaux a été vérifiée par nos soins [2] en utilisant une microsonde électronique et des observa- tions au microscope à balayage. Ces techniques ont permis de choisir les échantillons les plus homogènes en rejetant certains monocristaux possédant en inclusion du zinc hexagonal. Les monocristaux sont ensuite découpés sous forme de parallélépipèdes rectangles de surface utile de quelques $\mathrm{mm}^{2}$. Les faces en contact avec l'électrolyte sont orientées suivant les plans $\langle 110\rangle$ et $\langle 100\rangle$; la face cristalline est identifiée par la technique de Laue en retour [2];

- la réalisation du collecteur de courant est effectuée en utilisant la méthode de Aven [3] et Baker [4] de manière à obtenir sur la face arrière du cristal un contact peu résistif dont le comportement est parfaitement ohmique. Pour chaque électrode, nous avons vérifié l'ohmicité des contacts sous polarisation [5]. L'électrode munie de son contact est placée dans un dispositif d'électrode à disque tournant dont l'originalité consiste à permettre une observation physique de l'électrode avant et après le traitement électrochimique sans détruire le matériau [5]. Des vitesses de rotation de l'électrode sont possibles entre $10 \mathrm{rd}^{-\mathrm{s}^{-1}}$ et $300 \mathrm{rd} \cdot \mathrm{s}^{-1}$ avec une excentricité de l'ensemble tournant inférieure à $2 \%$;

- les surfaces en contact avec la solution n'étant pas natives, il est nécessaire de les activer. Le traite- 
ment se fait en deux étapes :- le mạtériau subit tout d'abord un polissage mécanique sur feutres doux à l'aide de pâtes diamant de grains différents (entre $20 \mu \mathrm{m}$ et $0,25 \mu \mathrm{m}$ ) puis après un lavage sous ultrasons, la couche écrouie est éliminée juste avant l'expérience par un décapage chimique pendant quelques secondes dans une solution de méthanol bromé à $2 \%$. La surface prend alors une couleur rouge rubis;

- l'électrode est placée dans l'obscurité totale dans une cellule d'électrolyse contenant une électrode inattaquable (disque d'or pur de $5 \mathrm{~cm}$ de diamètre) sous atmosphère contrôlée (argon $\mathrm{U}$ ) à température ambiante $(T=293 \pm 1 \mathrm{~K})$. Les potentiels sont mesurés par rapport à une électrode de référence (sulfate mercureux à sulfate de potassium saturé, notée ESM) puis calculés par rapport à l'électrode normale à hydrogène (notée ENH).

2. Expérimentation. - Le comportement de l'électrode semiconductrice est étudié selon le principe suivant :

- après avoir plongé l'électrode dans un électrolyte indifférent de $\mathrm{pH}$ variable et suffisamment concentré $(C \geqslant 0,5 \mathrm{M})$, nous recherchons le domaine de stabilité électrochimique de l'électrode, c'est-à-dire le domaine de potentiel pour lequel l'électrode de part et d'autre de son potentiel de repos peut être considérée comme idéalement polarisable (l'intensité du courant doit rester très faible : $i \leqslant 0,1 \mu \mathrm{A} . \mathrm{cm}^{-2}$ ). Cette recherche est effectuée par la méthode de chronoampérométrie à variation linéaire de potentiel (notée CAV́LP) : l'obtention des courbes courant-potentiel est effectuée avec une vitesse de balayage en tension très lente ;

- les propriétés de l'interface électrode-solution sont alors obtenues en effectuant des mesures de l'impédance de la cellule dans le domaine d'idéale polarisation de l'électrode. Pour cela on impose à l'électrode simultanément une polarisation continue lentement et linéairement variable contrôlée par un potensiostat et une perturbation sinusoïdale de faible amplitude $(\tilde{v}=5 \mathrm{mV})$ et de fréquence variable. Le domaine de fréquences comprend une trentaine de valeurs réparties entre $83 \mathrm{~Hz}$ et $100 \mathrm{kHz}$. La mesure de l'impédance est obtenue à l'aide d'un système de détection synchrone de marque Brookdeal donnant simultanément la réponse en phase et en quadrature de phase avec la perturbation. La résistance des parties non perturbées de la solution (résistance d'électrolyte) et du solide (notée $R_{\mathrm{E}}$ ) est corrigée de l'impédance globale directement par l'intermédiaire d'un correcteur de chute ohmique.

L'amplitude du domaine de fréquences nécessite l'utilisation de deux montages électroniques différents [6] :

- entre $83 \mathrm{~Hz}$ et $2 \mathrm{kHz}$, on impose la perturbation sinusoïdale sous forme d'une tension; on mesure alors les projections du vecteur courant sur un axe en phase et sur un axe en quadrature de phase avec la tension. Les valeurs expérimentales obtenues sur un enregistreur XY à deux voies en $\mathrm{Y}$ sont directement proportionnelles à $1 / R_{\mathrm{p}}$ et à $\omega C_{\mathrm{p}}$ (Fig. $\left.1 a\right)$;

- au-delà de $2000 \mathrm{~Hz}$, la perturbation est imposée sous forme d'un courant; on mesure les projections $\mathrm{du}$ vecteur tension sur les deux axes : les valeurs expérimentales sont proportionnelles à $R_{\mathrm{s}}$ et à $1 / \omega C_{\mathrm{s}}$ (Fig. 1b).

Un système d'acquisition de données directement raccordé à un miniordinateur permet une analyse rapide des résultats. En particulier le calcul des grandeurs $R_{\mathrm{s}}, R_{\mathrm{p}}, C_{\mathrm{s}}, C_{\mathrm{p}}, C_{\mathrm{p}}^{-2}$ après corrections de $R_{\mathrm{E}}$ [7] est obtenu avec une précision de $1 \%$ pour chaque valeur du potentiel. Les courbes $C_{\mathrm{p}}^{-2}=f(V)$ sont directement obtenues sur une table traçante Hewlett Packard.

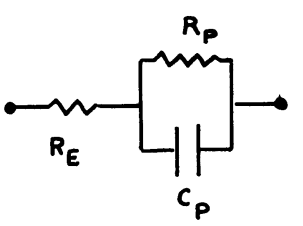

(a)

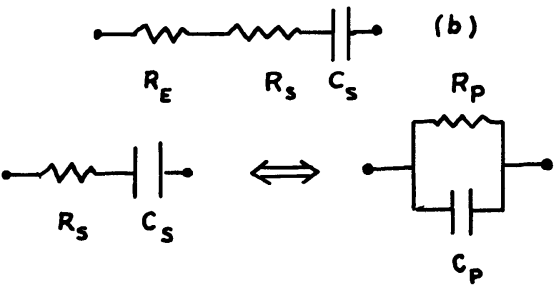

(a)

$$
Y=\frac{1}{Z}=\frac{1}{R_{P}}+j \omega C_{P}
$$$$
i=\frac{v}{Z} \stackrel{C_{p}}{\sim \frac{1}{R_{p}}}
$$

$$
z=R_{s}-j \frac{1}{\omega C_{s}}
$$

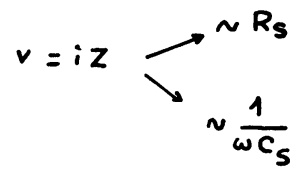

Fig. 1. - Schéma électrique équivalent à l'interface : a) potentiostatique, $b$ ) galvanostatique.

[Scheme equivalent to the interface.]

3. Résultats. - La recherche du domaine de stabilité de l'électrode est effectuée dans différents milieux et pour les deux orientations cristallographiques. La jonction $\mathrm{ZnTe}$-électrolyte possède des propriétés rectifiantes (Fig. 2). On observe un courant anodique important à partir d'un potentiel $V_{0}$. Ce courant est la conséquence de la dissolution anodique du cristal car l'oxydation des ions du solvant est impossible à ce potentiel. Le potentiel $V_{0}$ se déplaçant vers les polarisations négatives de $60 \mathrm{mV} / \mathrm{u} \mathrm{pH}$ lorsque le $\mathrm{pH}$ croît, la dissolution anodique fait intervenir les anions $\mathrm{OH}^{-}$. Pour la face cristalline la plus dense $(\langle 110\rangle)$, la dissolution anodique est repoussée de 


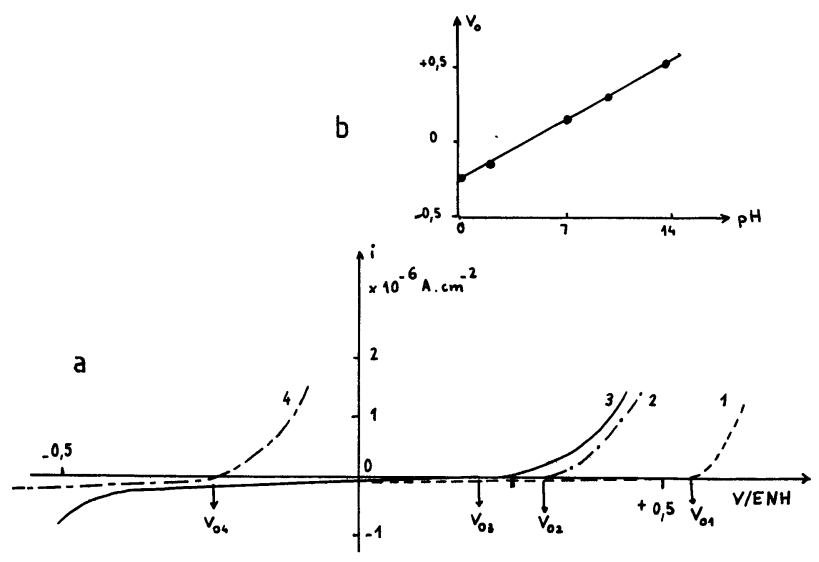

Fig. 2. $-a$ ) courbes courant tension $i=f(V): 1$. plan $\langle 100\rangle$ milieu acide; 2. plan $\langle 110\rangle$ milieu neutre; 3 . plan $\langle 100\rangle$ milieu neutre; 4. plan $\langle 100\rangle$ milieu basique; $b)$ variation du potentiel $V_{0}$ avec le $\mathrm{pH}$.

[Curves current voltage for different medium.]

$150 \mathrm{mV}$ vers les potentiels positifs. Le courant reste très faible dans un domaine de potentiel de l'ordre de $0,5 \mathrm{~V}$. En polarisation inverse il apparaît un courant cathodique; ce courant semble lié à la présence de défauts en surface et nous l'expliciterons plus tard. Lorsque l'on inverse le sens de la polarisation, si l'électrode ne tourne pas, on observe un phénomène d'hystérésis en courant. L'amplitude de cet hystérésis varie avec la vitesse de rotation de l'électrode tant que celle-ci reste inférieure à $20 \mathrm{rd}^{-\mathrm{s}^{-1}}$. Ceci implique une lente corrosion du matériau dans l'obscurité. Pour s'affranchir des problèmes concernant la cinétique de cette corrosion, toutes les mesures sont effectuées avec

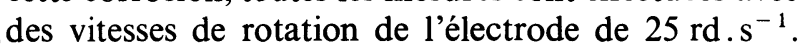
Dans ce cas si la vitesse du balayage en potentiel est suffisamment lente $\left(v \leqslant 1 \mathrm{mV}\right.$. $\left.\mathrm{s}^{-1}\right)$ on n'observe pas d'hystérésis sur les courbes d'impédance et il reste très faible pour les courbes courant-tension.

Dans le domaine de potentiel correspondant à l'idéale polarisation de l'électrode, les valeurs des éléments de l'impédance globale sont mesurées. Les valeurs de la résistance $R_{\mathrm{p}}$ et $C_{\mathrm{p}}$ du schéma électrique équivalant à l'interface (Fig. 1) sont obtenues en fonction du potentiel imposé à l'électrode. Pour chaque orientation on examine dans un milieu donné les deux composantes de cette impédance :

- les résistances et les capacités mesurées dépendent fortement de la fréquence angulaire $\omega$ de la perturbation. Pour un potentiel fixé, la capacité $C_{\mathrm{p}}$ décroît lorsque la fréquence croît. Les courbes $C_{\mathrm{p}}^{-2}=f(V)$ ne sont pas linéaires pour une fréquence donnée (Fig. 3). $C_{\mathrm{p}}$ ne représente donc pas la capacité de charge d'espace du semiconducteur selon le schéma de Brattain [8]. La résistance $R_{\mathrm{p}}$ décroît à potentiel constant lorsque la fréquence $F$ croît et à fréquence fixe croît rapidement avec le potentiel. Ces résultats présentés à propos de l'orientation $\langle 100\rangle$ en milieu basique se retrouvent pour le plan $\langle 110\rangle$ et dans tous

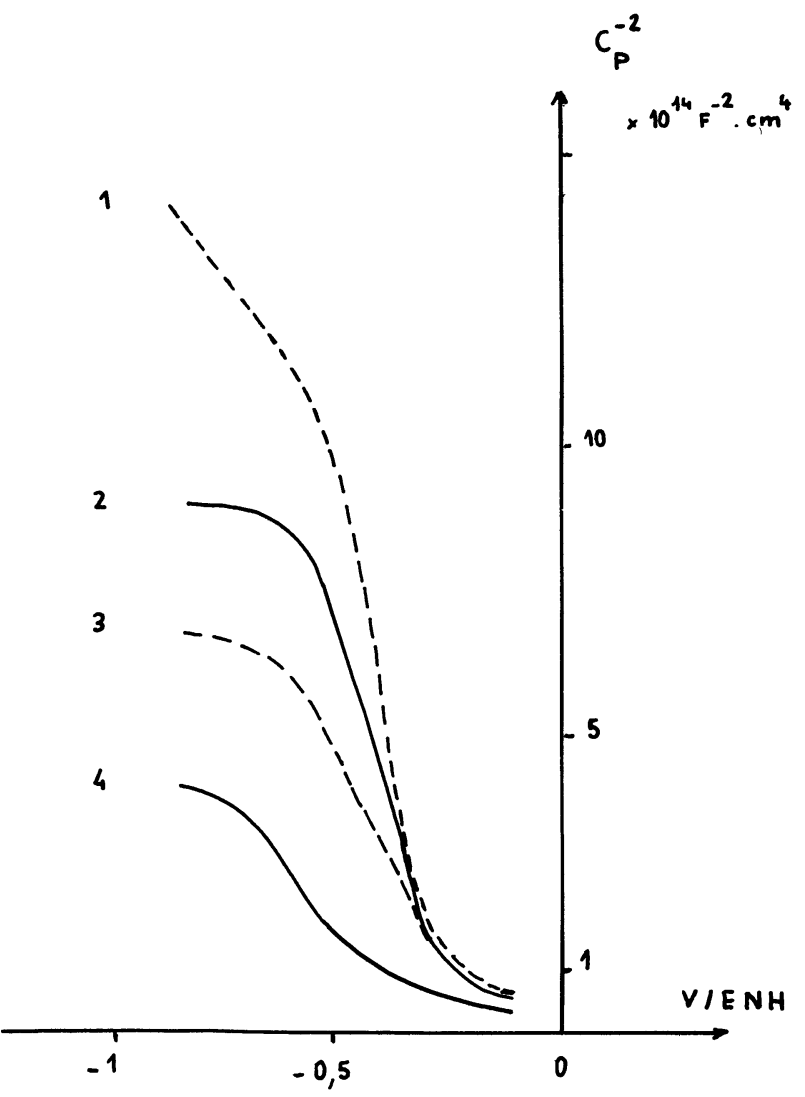

Fig. 3. - Courbes $C_{\mathrm{p}}^{-2}=f(V)$ plan $\langle 100\rangle$ milieu basique : 1. $F=100 \mathrm{kHz}$; 2. $F=80 \mathrm{kHz}$; $3 . F=50 \mathrm{kHz} ; 4 . F=10 \mathrm{kHz}$.

[Experimental Schottky plots $C_{\mathrm{p}}^{-2}=f(V)$ : basic medium.]

les milieux étudiés. Les éléments de l'impédance sont mesurés pour une surface de $1 \mathrm{~cm}^{2}$.

4. Discussion des résultats. - L'interface électrodesolution ne se comporte pas comme une jonction de type Schottky. Le schéma électrique équivalant à l'interface (Fig. 1) n'est pas utilisable car la capacité $C_{\mathrm{p}}$ ne peut en aucun cas s'identifier à la capacité de charge d'espace du semiconducteur $C_{\mathrm{CE}}$.

Les capacités varient avec la fréquence et la polarisation; à hautes fréquences (si $F \geqslant 10 \mathrm{kHz}$ ) les capacités $C_{\mathrm{p}}$ mesurées sont proportionnelles à $\omega^{-2}$, si le potentiel de l'électrode correspond au domaine d'idéale polarisation. Quant aux résistances séries $R_{\mathrm{s}}$, dans le même domaine de fréquences, elles augmentent exponentiellement avec le potentiel. La source de cette dispersion avec la fréquence des éléments de l'impédance doit être déterminée; seuls des effets ayant pour siège l'électrolyte, le sein du semiconducteur ou l'interface elle-même peuvent modifier le schéma électrique précédent.

L'impédance de la couche double de l'électrolyte peut modifier sous certaines conditions le comportement des capacités mesurées; dans le cas d'électrolytes indifférents concentrés, seule intervient la capacité de la couche d'Helmholtz $C_{\mathrm{H}}$ qui est indépendante 


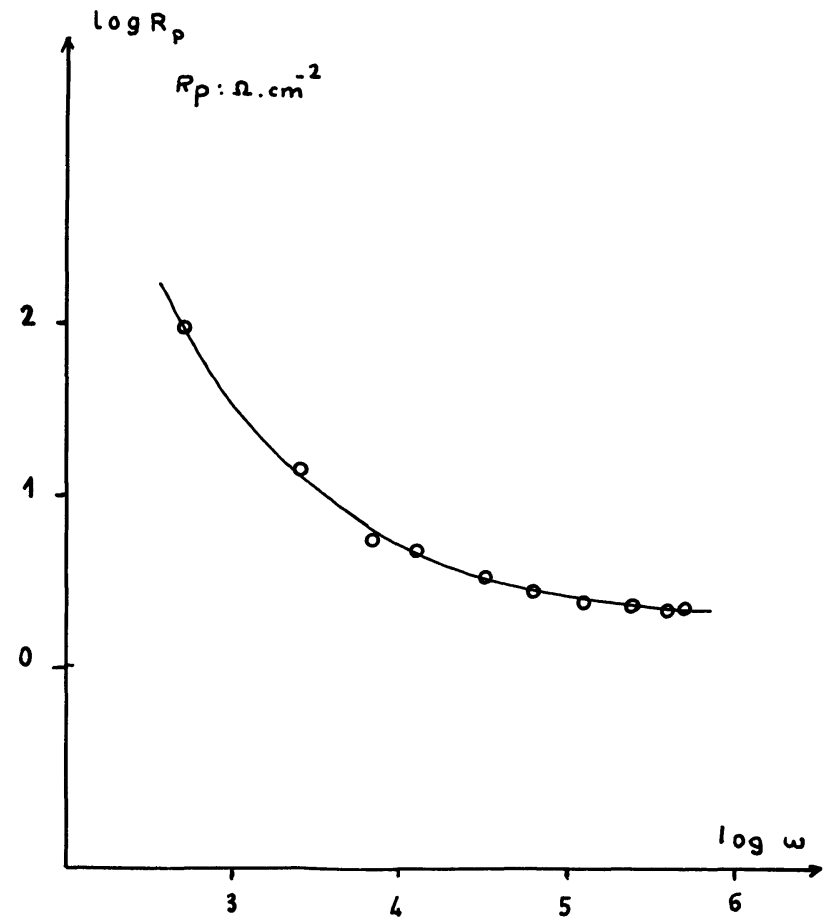

Fig. 4. - Variation des résistances $R_{\mathrm{p}}$ avec la fréquence $\omega$ plan $\langle 100\rangle$ milieu neutre.

[Variation of resistance $R_{\mathrm{p}}$ with the frequency $\omega$.]

de la fréquence et qui ne varie pas avec la polarisation tant qu'une adsorption spécifique des espèces n'intervient pas. Même dans ce cas la capacité $C_{\mathrm{H}}$ ne perturbe pas les mesures car associée en série avec la capacité de charge d'espace, sa valeur reste bien supérieure à $C_{\mathrm{CE}}\left(C_{\mathrm{H}} \geqslant 10 \mu \mathrm{F} . \mathrm{cm}^{-2}\right)$ [9]. L'impédance correspondante à haute fréquence est alors négligeable. L'action d'un processus faradique résiduel ainsi que la diffusion des espèces à l'électrode est également à rejeter car ces phénomènes ne peuvent expliquer les variations des capacités et des résistances avec la fréquence [10].

$\mathrm{Au}$ sein du semiconducteur par contre, la diffusion des porteurs minoritaires vers la surface (ici les électrons) peut aboutir à une variation de la capacité avec la fréquence proportionnelle à $\omega^{-2}$. Mais en aucune façon on n'observera dans ce cas une augmentation exponentielle de la résistance $R_{\mathrm{s}}$ avec la polarisation. De plus le tellurure de zinc est un semiconducteur à large bande interdite possédant une longueur de diffusion des porteurs minoritaires élevée. L'effet dû à la diffusion de ces porteurs que nous avons évalué à l'aide du calcul théorique de Pleskov [10] reste alors négligeable.

Les effets observés sont donc caractéristiques d'un phénomène d'interface. Il faut envisager l'existence de niveaux électroniques localisés à la surface de l'électrode, niveaux liés à l'existence de la jonction et à la nature du matériau.

En présence d'états en surface la réponse de la cellule à une perturbation sinusoïdale est complexe.
L'étude théorique a été effectuée par Berz [11] et Gurevich [12]. Nous reprendrons ici leurs hypothèses. La position des états localisés en surface joue un rôle considérable dans la réalisation du transfert de charges qui a lieu soit avec la bande de conduction soit avec la bande de valence. Pour chacune de ces possibilités l'état de surface considéré intervient par un temps de relaxation propre. La réponse à la perturbation sinusoïdale est une impédance constituée par une capacité ${ }_{\mathrm{s}} C_{\mathrm{ES}}$ associée en série avec une résistance ${ }_{\mathrm{s}} R_{\mathrm{ES}}$. La constante de temps de l'état considéré est $t={ }_{\mathrm{s}} C_{\mathrm{ES}} \cdot \mathrm{s} R_{\mathrm{ES}}$. Cette impédance est en parallèle sur la capacité de charge d'espace selon le schéma électrique de la figure $5 a$. a

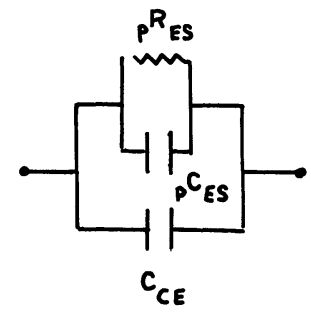

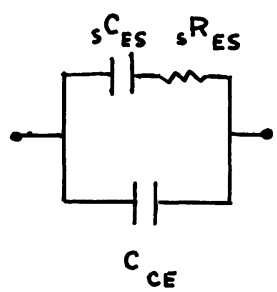

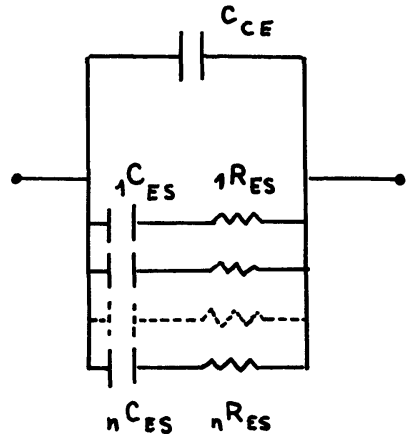

Fig. 5. - Schéma électrique équivalent : $a$ ) un seul type d'état en surface; $b$ ) plusieurs types d'état en surface.

[Scheme equivalent to the interface with surface states.]

L'impédance varie avec la fréquence de la perturbation selon l'expression :

$$
Z_{\mathrm{ES}}(\omega)=Z_{\mathrm{ES}}(0) \frac{1-i \omega t}{1+\omega^{2} t^{2}}
$$

Pour toute valeur de la polarisation, tant que $\omega$ n'a pas atteint la fréquence caractéristique de l'état considéré $\Omega=1 / t$, le terme capacitif reste constant ; au-delà, la capacité décroît avec la fréquence comme $\omega^{-2}$. Plusieurs niveaux d'énergies localisés à des potentiels différents peuvent exister. Mais si l'on tient compte du fait que la distribution des états d'énergie en surface obéit à la statistique de Fermi-Dirac, il faut tenir compte de l'éventualité de l'existence de plusieurs types d'états pour une même valeur du potentiel. Chaque type d'état est caractérisé par un temps de relaxation $t_{\mathrm{i}}$. Le schéma électrique équivalent est celui de la figure $5 b$. La densité énergétique des états a été prise en compte en supposant que pour un potentiel donné tous les états se trouvaient dans leur état 
de remplissage maximum. Si l'on tient compte de ces hypothèses la résistance série mesurée doit varier avec le potentiel selon une loi exponentielle.

Les variations de $R_{\mathrm{s}}$ avec le potentiel et de $C_{\mathrm{p}}$ avec la fréquence obéissant à des lois analogues à celles dues aux états en surface, nous avons extrapolé les capacités mesurées $C_{\mathrm{p}}$ à fréquence infinie et déterminé pour chaque valeur du potentiel la valeur $C_{\mathrm{p}}^{\prime}$ donnée par l'extrapolation des droites $C_{\mathrm{p}}=f\left(\omega^{-2}\right)$ à $\omega^{-2}=0$. Le schéma de la figure $5 b$ montre que cette valeur doit s'identifier à la capacité de charge d'espace du semiconducteur.

Les résultats obtenus sont conformes à la théorie ; les courbes $C_{\mathrm{p}}^{\prime-2}=f(V)$ sont linéaires (Fig. 6).

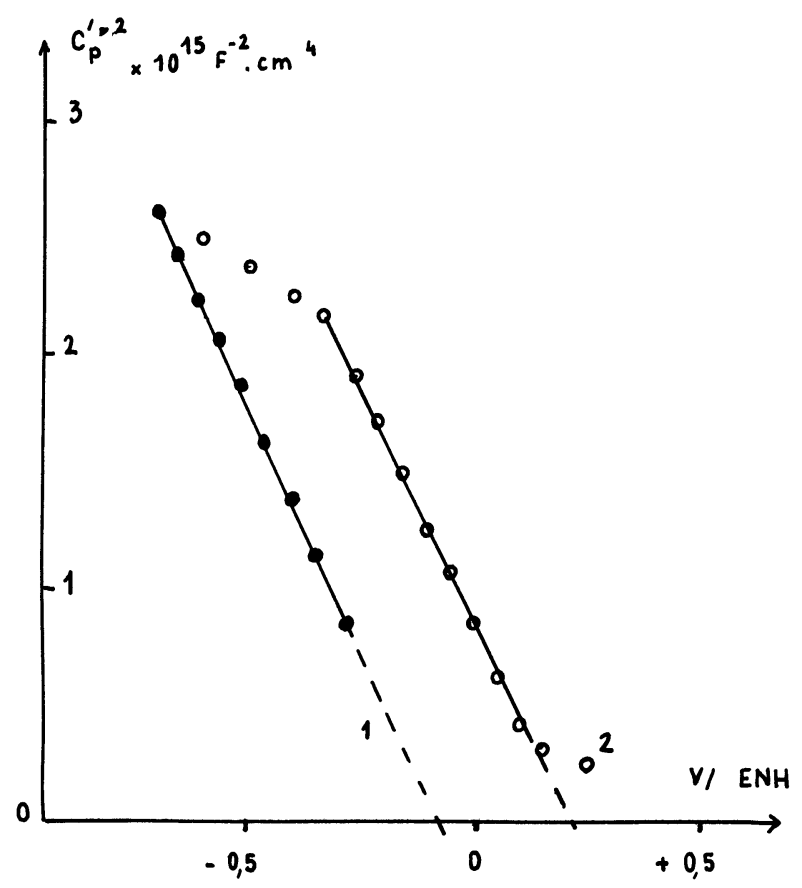

Fig. 6. - Courbes de Schottky : $C_{\mathrm{p}}^{\prime-2}=f(V): 1$. plan $\langle 100\rangle$ milieu basique ; 2 . plan $\langle 100\rangle$ milieu neutre.

[Schottky plots $C_{\mathrm{p}}^{\prime-2}=f(V)$.]

L'interface se comporte comme une diode Schottky. Nous avons alors comparé les expressions de $C_{\mathrm{p}}^{\prime}$ avec les valeurs théoriques calculées à partir des données physiques de la capacité de charge d'espace $C_{\mathrm{CE}}$ [10]. Une très bonne convergence des résultats est obtenue [13]. L'extrapolation pour $C_{\mathrm{p}}^{\prime-2}=0$ des droites obtenues en fonction de la polarisation dans différents milieux donne à $k T / e$ près le potentiel de bande plate du semiconducteur $\left(V_{\mathrm{BP}}\right)$. La pente de ces droites, indépendante du milieu électrolytique, est inversement proportionnelle au nombre de porteurs majoritaires du cristal que l'on peut ainsi calculer en supposant que tous les accepteurs sont ionisés; la valeur est :

$$
p_{0}=3,5 \times 10^{15} \mathrm{~cm}^{-3} .
$$

Elle est légèrement inférieure à celle obtenue par effet Hall [14].
La position du potentiel de bande plate varie avec le $\mathrm{pH}$ du milieu; le comportement du tellurure de zinc n'est pas Nernstien car la variation de $V_{\mathrm{BP}}$ avec le $\mathrm{pH}$ n'est que de $30 \mathrm{mV} / \mathrm{u} \mathrm{pH}$. Ce déplacement est évidemment lié à la nature des dipôles du solvant à la surface mais il est perturbé par la corrosion du cristal [15]. Si l'on charge l'électrode très négativement, c'est-àdire lors d'une polarisation très négative par rapport à $V_{\mathrm{BP}}$, on observe une variation brusque de la pente de la droite $C_{\mathrm{p}}^{\prime-2}=f(V)$. L'ionisation des porteurs de niveaux profonds intervient lorsque le potentiel atteint une certaine valeur [10]. La pente la plus faible doit correspondre au nombre total de porteurs ionisés, nombre très proche de celui mesuré par effet Hall : on obtient $p=8 \times 10^{15} \mathrm{~cm}^{-3}$ (par effet Hall : $p=1 \times 10^{16} \mathrm{~cm}^{-3}$ ). L'existence des niveaux accepteurs profonds a été montrée par ailleurs [16]. Ces niveaux peuvent être identifiés à des niveaux « $\mathrm{ZnO}$ ».

Lorsque l'index de la face cristalline augmente, le potentiel de bande plate se déplace pour un même milieu, vers les potentiels positifs. Ce déplacement est dû à la variation de la densité des atomes en surface entre les plans $\langle 100\rangle$ et $\langle 110\rangle$. Le potentiel de bande plate représentant la position du niveau de Fermi du semiconducteur par rapport à la référence ENH, cette variation correspond à la différence entre les travaux d'extraction pour les deux orientations. De plus la dissolution sélective des atomes de zinc et de tellure au cours du décapage crée des attaques préférentielles selon le plan et cela malgré le lavage sous ultrasons qui doit minimiser les dislocations du réseau en surface après polissage [17].

Les mesures d'impédance pour la jonction étudiée permettent également d'obtenir un certain nombre de renseignements sur les niveaux d'énergie localisés en surface. En effet, $C_{\mathrm{p}}-C_{\mathrm{p}}^{\prime}$ représente la somme des capacités dues aux différents états d'énergie présents pour un potentiel donné $\left(C_{\mathrm{p}}-C_{\mathrm{p}}^{\prime}={ }_{\mathrm{p}} C_{\mathrm{ES}}\right)$.

$$
\begin{aligned}
{ }_{\mathrm{p}} C_{\mathrm{ES}} & =\sum_{i}{ }_{\mathrm{p}} C_{\mathrm{ES}}^{i}=\sum_{i}{ }_{\mathrm{s}} C_{\mathrm{ES}}^{i} \cdot \frac{1}{1+\gamma_{\mathrm{i}}^{2}} \\
\gamma_{\mathrm{i}} & ={ }_{\mathrm{s}} R_{\mathrm{ES} \cdot \mathrm{s}}^{i} C_{\mathrm{ES}}^{i} \cdot \omega .
\end{aligned}
$$

Les courbes $C_{\mathrm{p}}-C_{\mathrm{p}}^{\prime}=f(\omega)$ sont représentées à la figure 7 ; elles présentent toutes le même aspect. En supposant que pour un potentiel donné il existe plusieurs niveaux dans leur état de remplissage maximum, il est possible de calculer le nombre d'éta ts présent et de déterminer une valeur approchée de leur constante de temps. Nous avons réalisé cela par simulation grâce à l'ordinateur et montré l'existence pour chaque potentiel de deux types d'états; les fréquences caractéristiques ont été calculées. Bien que le calcul ne soit qu'approché, l'existence de deux types d'états est confirmée par l'étude des termes résistifs $R_{\mathrm{p}}$ de l'impédance; ainsi que l'a montré Gomes [18], si un seul type d'état de surface existe, les courbes

$$
R_{\mathrm{p}}^{-1}=f\left(C_{\mathrm{p}}-C_{\mathrm{p}}^{\prime}\right)
$$




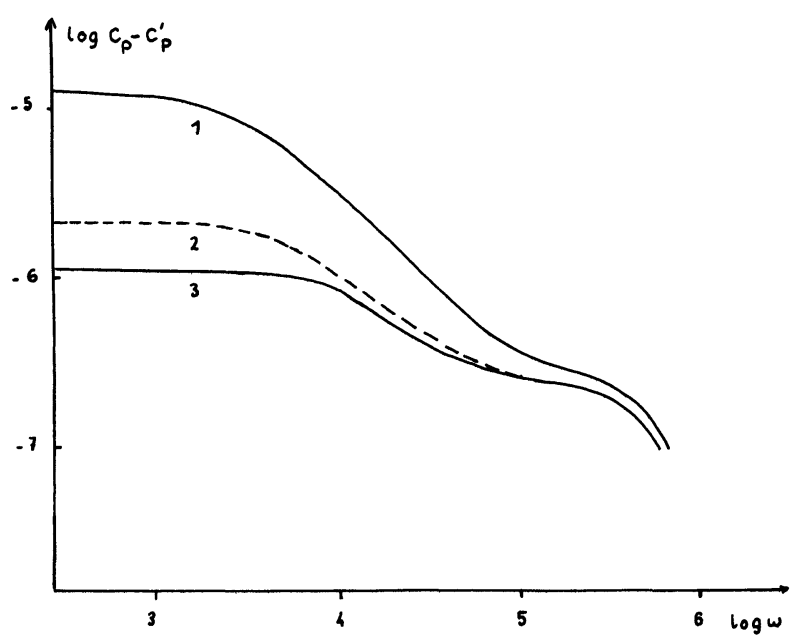

Fig. 7. - Capacités dues aux états en surface; variation avec $\omega$ :

1. $V=-0,55 \mathrm{~V} / \mathrm{ENH}$ )

2. $V=-0,75 \mathrm{~V} /$ ENH $\}$ plan $\langle 100\rangle$ milieu basique .

3. $V=-0,85 \mathrm{~V} / \mathrm{ENH}$

[Capacity due to surface states $C_{\mathrm{p}}-C_{\mathrm{p}}^{\prime}=f(\omega)$.]

doivent être linéaires. Dans notre cas, comme le montre la figure 8 , nous devons tenir compte de plus d'un état; la précision des mesures ne permet pas de différencier leur nombre.

Les temps de relaxation rapides sont indépendants du milieu support. Ce n'est plus le cas pour les temps

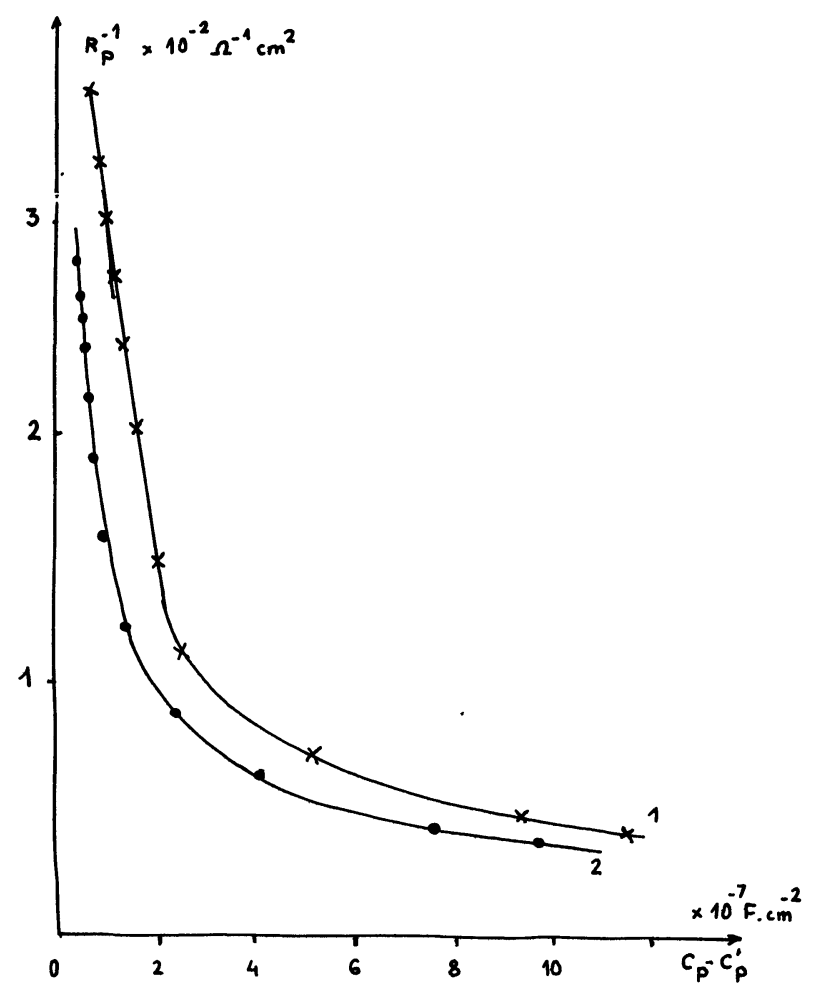

Fig. 8. - Variation $R_{\mathrm{p}}^{-1}=f\left(C_{\mathrm{p}} f C_{\mathrm{p}}^{\prime}\right)$ : plan $\langle 100\rangle$ milieu neutre : 1. $V=+0,2 \mathrm{~V} / \mathrm{ENH} ; 2 . V=-0,05 \mathrm{~V} / \mathrm{ENH}$.

[Variation of $R_{\mathrm{p}}^{-1}$ with $C_{\mathrm{p}}-C_{\mathrm{p}}^{\prime}$.] de relaxation lents qui dépendent du $\mathrm{pH}$. L'adsorption des anions du support perturbe donc l'existence et surtout l'état de remplissage de ces niveaux.

Les courbes $C_{\mathrm{p}}-C_{\mathrm{p}}^{\prime}=f(V)$ pour une fréquence donnée présentent un comportement analogue quelle que soit la fréquence (Fig. 9). Ces courbes impliquent l'existence d'un continuum d'états localisés dans un large domaine de potentiel correspondant à l'intérieur de la bande interdite du semiconducteur.

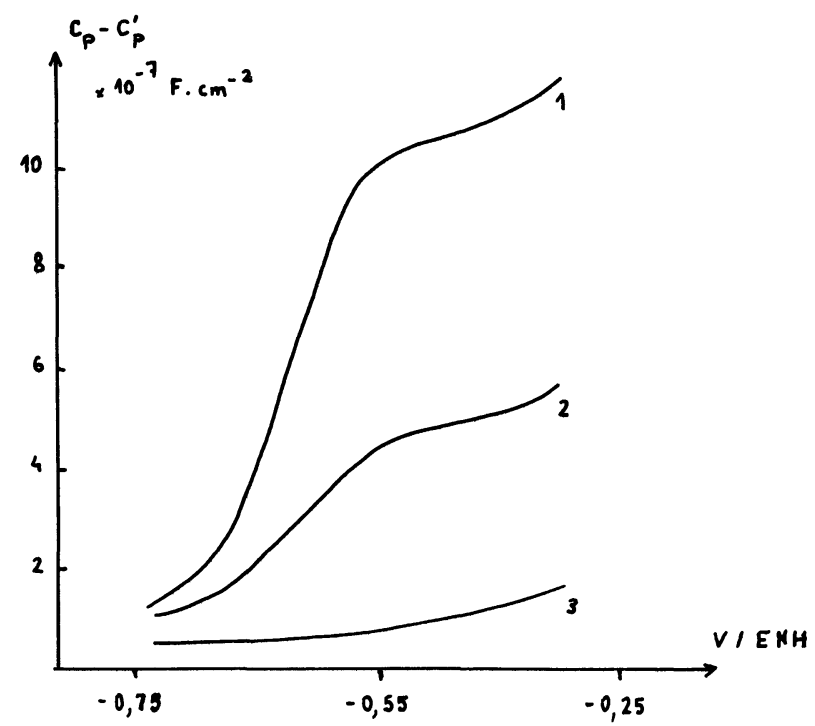

Fig. 9. - Variation des capacités $C_{\mathrm{p}}-C_{\mathrm{p}}^{\prime}$ avec la polarisation : 1. $F=100 \mathrm{~Hz}$; $2 . F=1000 \mathrm{~Hz}$; 3. $F=5000 \mathrm{~Hz}$.

[Variation of capacity $C_{\mathrm{p}}-C_{\mathrm{p}}^{\prime}$ with potential.]

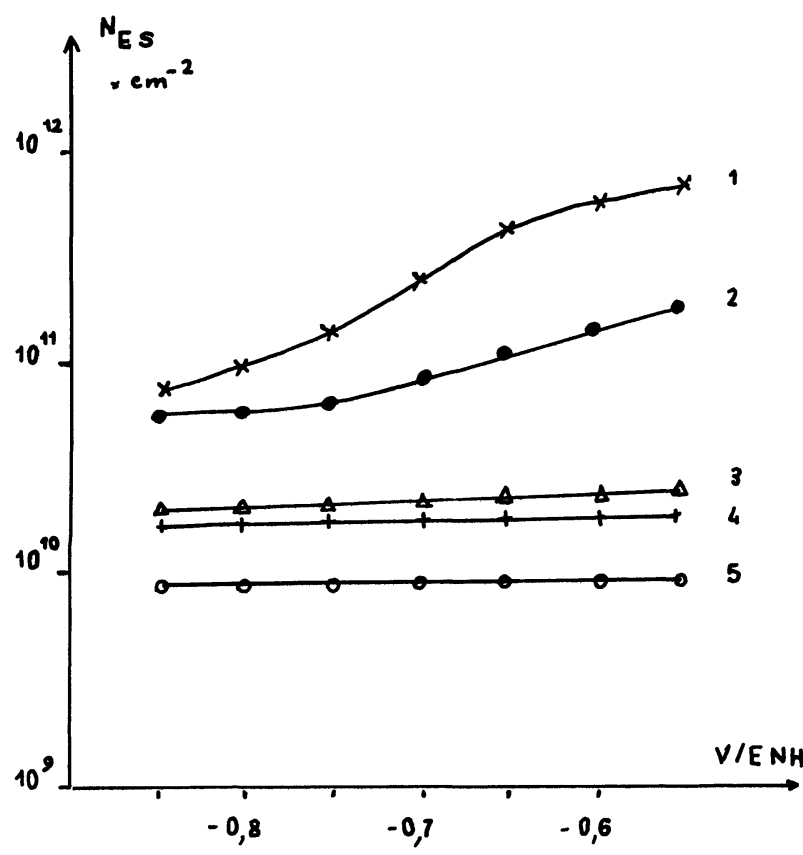

Fig. 10. - Nombre d'états en surface $N_{\mathrm{ES}}$ en fonction du potentiel. Milieu basique : $1 . \omega=100 ; 2 . \omega=1000 ; 3 . \omega=5000 ; 4$. $\omega=50000$; 5. $\omega=150000$.

[Variation of the number of surface states with the polarisation.] 
Ces états répartis près du potentiel de bande plate dans la bande interdite pourront jouer un grand rôle lors de la cinétique des réactions de transfert de charge à l'électrode.

La détermination par le calcul des capacités $C_{\mathrm{ES}}$ pour chaque type d'état permet l'évaluation de leur nombre $N_{\mathrm{ES}}[6]$. Ainsi que le montre la figure 10, le nombre de ces états oscille entre $10^{10}$ et $10^{11} \mathrm{~cm}^{-2}$. Ce nombre diminue lorsque la fréquence croît conformément aux prévisions théoriques [10]. A basse fréquence, il diminue lorsque la polarisation devient plus négative. Cela confirme l'influence de l'adsorption des anions de l'électrolyte.

5. Conclusion. - Ce travail, par les mesures de l'impédance de l'interface électrode-solution dans un large domaine de fréquences, a permis de connaître avec précision le comportement de cette interface. Nous avons ainsi montré l'existence de niveaux localisés à la surface et pu évaluer leur nombre et leur répartition énergétique.
A hautes fréquences, le comportement de type Schottky a été retrouvé et la mesure du nombre de porteurs du cristal déterminé. De plus l'obtention du potentiel de bande plate à permis le positionnement des niveaux d'énergie du matériau $\left(E_{\mathrm{C}}\right.$ et $\left.E_{\mathrm{v}}\right)$ par rapport à la référence ENH. Ces mesures sont indispensables pour l'étude des réactions électrochimiques de transfert de charges car elles conditionnent les possibilités d'échanges électroniques entre le semiconducteur et le système redox. Les résultats obtenus permettent d'envisager l'utilisation de ce matériau en cinétique électrochimique car l'existence des niveaux en surface devrait influencer considérablement la vitesse des réactions à l'électrode. Les mesures effectuées dans le noir ont été reprises en illuminant l'électrode. Les potentiels de bande plate alors obtenus confirment les résultats précédents.

Remerciements. - Je tiens à exprimer mes remerciements aux chercheurs du laboratoire de physique des solides du C.N.R.S. de Bellevue pour la préparation des monocristaux et pour les mesures d'effet Hall.

\section{Bibliographie}

[1] El Axadd et Rodot, M., C. R. Hebd. Séan. Acad. Sci. 272 (1971) 680.

[2] Sculfort, J. L. et Peslerbe, G., Résultats non publiés.

[3] Aven, M. et Garwacki, W., J. Electrochem. Soc. 114 (1967) 1063.

[4] Baker, W. D. et Milnes, A. E., J. Electrochem. Soc. 119 (1972) 1269.

[5] Sculfort, J. L., Thèse doctorat Paris (1976).

[6] Lemasson, P., Baticle, A. M. et Vennereau, P., Surf. Sci. 59 (1976) 177.

[7] Sculfort, J. L. et Baticle, A. M., C. R. Hebd. Séan. Acad. Sci. C 288 (1979) 109.

[8] Brattain, W. H. et Boddy, P. J., J. Electrochem. Soc. 109 (1962) 574.

[9] Pettinger, B., Schoeppel, H. R., Yokohà̄a, T. et Gerischer, H., Ber. Bunsenges. Phys. Chem. 79 (1975) 1206.
[10] Pleskov, Yu. et Myamlin, V. A., Electrochemistry of semiconductors (Plenum Press, N. Y.) 1965.

[11] Berz, F., J. Electrochem. Soc. 6 (1959) 197.

[12] Gurevich, Yu. et Myamlin, V. A., Izv. Akad. NAUK CCCP (1964) 1778.

[13] Sculfort, J. L. et Baticle, A. M., Elektrokhimiya 14 (1978) 872.

[14] El AKaAD, Communication personnelle.

[15] Gomes, W. P., Van Wezemael, A. M., Laflere, W. H. et Cardon, F., J. Electroanalytical Chem. 87 (1978) 105.

[16] Al DaAl, Thèse doctorat Paris (1975).

[17] Buck, J. M. et Mac KIM, F. S., Phys. Rev. 106 (1957) 904.

[18] Gomes, W. P. et Cardon, F., Ber. Bunsenges. Phys. Chem. 74 (1970) 431 